\title{
Block diagonalisation-based multiuser multiple input multiple output-aided downlink relaying
}

\author{
W. Liu $^{1}$ C. $L i^{2} \quad$ J.-D. $L i^{1} \quad$ L. Hanzo $^{3}$ \\ ${ }^{1}$ State Key Labs of ISN, Xidian University, Xian, Shaanxi 710071, People's Republic of China \\ ${ }^{2}$ School of Computer and Technology, Xi'an University of Posts and Telecommunications, Xian, Shaanxi 710061, \\ People's Republic of China \\ ${ }^{3}$ School of ECS, University of Southampton, Southampton, SO17 1BJ, UK \\ E-mail: Ih@ecs.soton.ac.uk
}

\begin{abstract}
A novel block diagonalisation (BD)-based Multiuser multiple input multiple output (MU-MIMO)-aided relaying scheme is proposed for downlink transmissions, which does not require any channel state information at the base station (BS) and decomposes a MU-MIMO-aided relaying system into several parallel single-user MIMO-assisted relaying schemes. Furthermore, based on the proposed algorithm the optimal linear processing matrix designed for the maximum achievable capacity is derived, which significantly outperforms the so-called naive weighting matrix.
\end{abstract}

\section{Nomenclature \\ $\boldsymbol{x}_{k}$ Symbol vector transmitted to the $k$ th MS from BS \\ $\boldsymbol{H} \quad$ Channel matrix between the BS and the RS \\ $\boldsymbol{H}_{k} \quad$ Channel matrix used for $k$ th MS between the BS and the RS \\ $y_{r} \quad$ Received signal at the RS \\ $\boldsymbol{W} \quad$ Weighting matrix at the RS \\ $\boldsymbol{x}_{r} \quad$ Transmitted signal at the RS \\ $\boldsymbol{T}_{k} \quad$ Postprocessing matrix for the $k$ th MS at the RS \\ $\boldsymbol{P}_{k} \quad$ Preprocessing matrix for the $k$ th MS at the RS \\ $\boldsymbol{F}_{k} \quad$ Weighting matrix for the $k$ th MS at the RS \\ $\boldsymbol{x}_{k} \quad$ Transmitted signal for the $k$ th MS at the RS \\ $\tilde{\boldsymbol{H}}_{k} \quad$ Channel matrix between the RS and the $k$ th MS \\ $\tilde{\boldsymbol{y}}_{k} \quad$ Received signal at the $k$ th MS}

\section{Introduction}

High-rate wireless transmissions can be supported by multiple antennas employed both at the base station (BS) and the mobile stations (MS), when using the concept of multiple input multiple output (MIMO) systems, since they are capable of substantially increasing the achievable system capacity [1]. Hence diverse MIMO-aided transmission schemes have been developed. In [2], the socalled Bell Laboratories layered space-time (BLAST) MIMO system was proposed, which is capable of achieving a beneficial multiplexing gain. By contrast, space time block codes are capable of attaining a diversity gain [3]. In order to achieve the maximum attainable throughput, a BLAST-based MIMO system is adopted in this paper.

As a design alternative, relay-assisted transmission techniques also attracted substantial research interests, since they are capable of achieving a spatial diversity gain, hence extending the cellular coverage area, even when using a reduced transmitter power [4]. Relay strategies can be roughly divided into two categories, regenerative and nonregenerative. In a regenerative scenario the relay decodes the data and then re-encodes the symbols before their retransmission. By contrast, non-regenerative relays linearly process the received signal and forward it. The inherent drawback of the regenerative relay is the increased complexity imposed by the employment of sophisticated decoding algorithms. Furthermore, decoding at the relay station (RS) may also result in a potential 'eavesdropping' problem. For these reasons a non-regenerative relay is considered in this paper.

Single-user MIMO-aided relaying has been investigated in [5-9]. Specifically, the system performance may be significantly improved by employing an optimal linear processing matrix at the RS, which may be designed using different optimisation criteria, such as for example that of achieving the maximum capacity [7], or the minimum mean square error [8]. We assume that only the RS and the MS have the downlink (DL) channel state information (CSI) for the link spanning from the BS to RS and from the RS to MS, but not the BS. Hence no CSI is fed back from the RS to $\mathrm{BS}$ and $\mathrm{MS}$ to $\mathrm{BS}$.

In multiuser MIMO-aided DL relaying most research concentrated on the scenario, where only a single receive antenna is used at each MS [10, 11]. Furthermore, it was typically assumed that the BS knows the DL CSI [10, 11]. However, it would be rather challenging to inform the BS 
of the CSI between the RS and each MS, especially for highvelocity MSs. It would be a slightly more realistic scenario for the RS to estimate the CSI for the link spanning from the BS to the RS and that between the RS and each MS [7, 8].

Against this background, we proposed a block diagonalisation (BD)-based MIMO-aided multiuser relaying scheme employing linear matrix-multiplication assisted processing at the RS for decomposing this multiuser scheme into low-complexity single-user ones. Furthermore, based on the proposed algorithm, the optimum linear processing matrix yielding the maximum achievable capacity is derived.

The paper is structured as follows. In Section 2, the MIMO-aided relaying assisted multiuser system employing matrix-multiplication-based linear processing at the RS is discussed. In Section 3 BD-based linear preprocessing is used at the RS. In Section 4 the canonical form of the linear processing matrix designed for attaining the maximum achievable capacity is derived and the maximum achievable capacity is quantified. Our simulation results are provided in Section 5, while our conclusions are offered in Section 6. Furthermore, in order to make it easier to understand the paper, some important symbols are listed in the nomenclature.

\section{Multiuser MIMO relay employing linear processing at the RS}

The multiuser MIMO-aided DL relaying scheme employing linear processing at the RS is shown in Fig. 1, which relies on a single BS using $M$ DL transmit antennas and a single RS equipped with $R$ transmit/receive antennas, as shown in Fig. 1. Furthermore, there are $K \mathrm{MSs}$, each having $N_{k}$ DL receive antennas.

Assume that the $M_{k}$-component symbol vector $\boldsymbol{x}_{k}$ has to be transmitted to the $k$ th $\mathrm{MS}$, which is given by

$$
\boldsymbol{x}_{k}=\left[x_{k 1}, x_{k 2}, \ldots, x_{k M_{k}}\right]^{\mathrm{T}}
$$

where the total number of symbols transmitted to each MS is identical to the number of BS antennas, yielding $\sum_{k=1}^{K} M_{k}=M$. The BS's signal vector $\boldsymbol{x}$ transmitted in the DL to the $K$ MSs may be expressed as

$$
\boldsymbol{x}=\left[\boldsymbol{x}_{1}^{\mathrm{T}}, \ldots, \boldsymbol{x}_{K}^{\mathrm{T}}\right]^{\mathrm{T}}
$$

Furthermore, we assume that the DL channel between the BS and the RS experiences frequency-flat fading, which is described by a $(R \times M)$-dimensional matrix $\boldsymbol{H}$. The signal $\boldsymbol{y}_{r}$ received at the RS is given by

$$
\boldsymbol{y}_{r}=\boldsymbol{H} \boldsymbol{x}+\boldsymbol{n}=\sum_{i=1}^{K} \boldsymbol{H}_{i} \boldsymbol{x}_{i}+\boldsymbol{n}
$$

where $\boldsymbol{H}_{i}$ is an $\left(R \times M_{k}\right)$-dimensional matrix, representing the channel coefficients between the specific $M_{k}$ transmit antennas of the BS which are dedicated to the $k$ th MS, and the $R$ receive antennas at the RS, while $\boldsymbol{n}$ is a length- $R$ noise observation vector, which is assumed to be Gaussian distributed with a zero mean and a covariance matrix of $\sigma_{1}^{2} \boldsymbol{I}_{R}$.

As shown in Fig. 1, an $(R \times R)$-dimensional linear processing matrix $\boldsymbol{W}$ is used at the RS, hence the signal transmitted by the RS is expressed as

$$
\boldsymbol{x}_{r}=\boldsymbol{W} \boldsymbol{y}_{r}=\boldsymbol{W} \sum_{i=1}^{K} \boldsymbol{H}_{i} \boldsymbol{x}_{i}+\boldsymbol{W} \boldsymbol{n}
$$

The $N_{k}$-component received signal vector $\tilde{y}_{k}$ of the $k$ th MS is given by

$$
\begin{aligned}
\tilde{\boldsymbol{y}}_{k} & =\tilde{\boldsymbol{H}}_{k} \boldsymbol{x}_{r}+\tilde{\boldsymbol{n}}_{k} \\
& =\tilde{\boldsymbol{H}}_{k} \boldsymbol{W} \boldsymbol{H}_{k} \boldsymbol{x}_{k}+\tilde{\boldsymbol{H}}_{k} \boldsymbol{W} \sum_{i=1, i \neq k}^{K} \boldsymbol{H}_{i} \boldsymbol{x}_{i}+\tilde{\boldsymbol{H}}_{k} \boldsymbol{W} \boldsymbol{n}+\tilde{\boldsymbol{n}}_{k}
\end{aligned}
$$

where the $\left(N_{k} \times R\right)$-dimensional matrix $\tilde{\boldsymbol{H}}_{k}$ represents the channel coefficients between the RS and the $k$ th MS. Furthermore, similarly to the BS-RS link, a flat-fading channel is assumed also for the RS-MS link, while $\tilde{\boldsymbol{n}}_{k}$ is a length- $N_{k}$ noise observation vector at the $k$ th $\mathrm{MS}$, which is assumed to be Gaussian distributed with a zero mean and a covariance matrix of $\sigma_{2}^{2} \boldsymbol{I}_{N_{k}}$. As we can see from the second term of (5), the multiuser interference (MUI) imposed on the $k$ th MS is given by $\sum_{i=1, i \neq k}^{K} \tilde{\boldsymbol{H}}_{k} \boldsymbol{W} \boldsymbol{H}_{i} \boldsymbol{x}_{i}$.

By appropriately choosing the RS's weight-matrix $\boldsymbol{W}$, the MUI can be mitigated for the $k$ th MS. In the next section, we determine the BD-based linear processing matrix $\boldsymbol{W}$ of the RS.

\section{BD-based linear processing matrix at the RS}

Again, the MUI imposed on the $k$ th MS's received signal $\boldsymbol{y}_{r}$ at the RS is given by $\sum_{i=1, i \neq k}^{K} \boldsymbol{H}_{i} \boldsymbol{x}_{i}$. In order to remove the MUI imposed on the $k$ th MS's signal at the RS, we invoke the postprocessing matrix $\boldsymbol{T}_{k}$ of Fig. 1 at the RS, resulting in
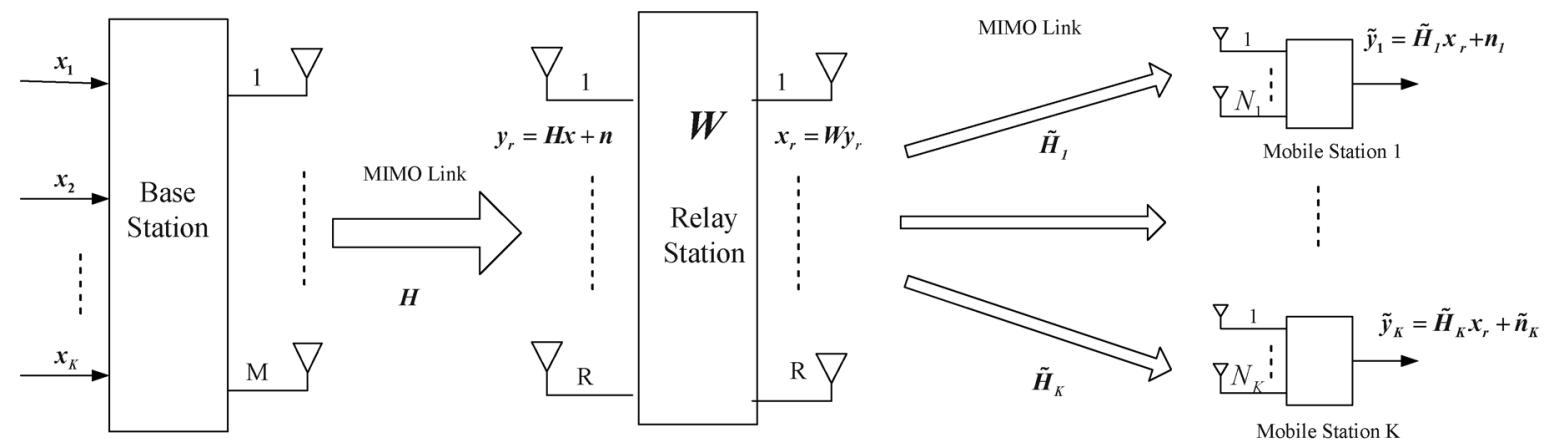

Fig. 1 Schematic representation of the multiuser MIMO relay system with linear processing at RS and multiple antenna at each mobile 
the postprocessed vector $\hat{\boldsymbol{y}}$ given by

$$
\hat{\boldsymbol{y}}_{k}=\boldsymbol{T}_{k} \boldsymbol{y}_{r}=\boldsymbol{T}_{k} \boldsymbol{H}_{k} \boldsymbol{x}_{k}+\boldsymbol{T}_{k} \sum_{i=1, i \neq k}^{K} \boldsymbol{H}_{i} \boldsymbol{x}_{i}+\boldsymbol{T}_{k} \boldsymbol{n}
$$

Furthermore, let us define an $\left(R \times \sum_{i=1, i \neq k}^{K} M_{i}\right)$ - component matrix $\hat{\boldsymbol{H}}_{k}$ as

$$
\hat{\boldsymbol{H}}_{k}=\left[\boldsymbol{H}_{1}, \ldots, \boldsymbol{H}_{(k-1)}, \boldsymbol{H}_{(k+1)}, \ldots, \boldsymbol{H}_{K}\right]
$$

Moreover, let us assume $R>\max \left(\sum_{i=1, i \neq k}^{K} M_{i}\right)$, hence we have $\operatorname{rank}(\hat{\boldsymbol{H}}) \leq \sum_{i=1, i \neq k}^{K} M_{i}$. If the elements in each channel matrix are independent identically distributed (i.i.d), we have $\operatorname{rank}\left(\hat{\boldsymbol{H}}_{k}\right)=\sum_{i=1, i \neq k}^{K} M_{i}$ with a unity probability [12] and the singular value decomposition (SVD) of $\hat{\boldsymbol{H}}_{k}$ can be expressed as

$$
\hat{\boldsymbol{H}}_{k}=\left[\hat{\boldsymbol{U}}_{k s} \hat{\boldsymbol{U}}_{k n}\right]\left[\begin{array}{c}
\hat{\boldsymbol{\Lambda}}_{k}^{1 / 2} \\
\mathbf{0}
\end{array}\right] \hat{\boldsymbol{V}}_{k}^{H}, \quad k=1,2, \ldots, K
$$

where $\hat{\boldsymbol{\Lambda}}_{k}=\operatorname{diag}\left\{\lambda_{1}, \lambda_{2}, \ldots, \lambda_{\sum_{i=1, i \neq k}^{K} M_{i}}\right\}$ contains the $\sum_{i=1, i \neq k}^{K} M_{i}$ non-zero eigenvalues of $\hat{\boldsymbol{H}}_{k}^{\mathrm{H}} \hat{\boldsymbol{H}}_{k}$ or $\hat{\boldsymbol{H}}_{k} \hat{\boldsymbol{H}}_{k}^{\mathrm{H}}$. Furthermore, the $\left(R \times \sum_{i=1, i \neq k}^{K} M_{i}\right)$-component semiunitary matrix $\hat{\boldsymbol{U}}_{k s}$ consists of the $\sum_{i=1, i \neq k}^{K} M_{i}$ eigenvectors corresponding to the signal subspace of $\hat{\boldsymbol{H}}_{k} \hat{\boldsymbol{H}}_{k}^{\mathrm{H}}$, while the $\left(R \times R-\sum_{i=1, i \neq k}^{K} M_{i}\right)$-component semi-unitary matrix $\hat{\boldsymbol{U}}_{k s}$ $\hat{\boldsymbol{U}}_{k n}$ consists of the $\left(R-\sum_{i=1, i \neq k}^{K} M_{i}\right)$ eigenvectors corresponding to the null subspace of $\hat{\boldsymbol{H}}_{k} \hat{\boldsymbol{H}}_{k}^{\mathrm{H}}$. Similarly, the columns of $\hat{\boldsymbol{V}}_{k}$ correspond to the eigenvectors of $\hat{\boldsymbol{H}}_{k}^{\mathrm{H}} \hat{\boldsymbol{H}}_{k}$.

We can now specifically design the RS's $\left(L_{k} \times R\right)$ component postprocessing matrix $\boldsymbol{T}_{k}$, where we have $L_{k}=R-\sum_{i=1, i \neq k}^{K} M_{i}$, so that it removes the MUI from the $k$ th MS's signal at the RS, which is ensured by choosing $T_{k}$ as

$$
\boldsymbol{T}_{k}=\hat{\boldsymbol{U}}_{k n}^{\mathrm{H}}
$$

Upon substituting (9) into (6), we arrive at

$$
\hat{\boldsymbol{y}}_{k}=\hat{\boldsymbol{U}}_{k n}^{\mathrm{H}} \boldsymbol{y}_{r}=\hat{\boldsymbol{U}}_{k n}^{\mathrm{H}} \boldsymbol{H}_{k} \boldsymbol{x}_{k}+\boldsymbol{n}_{k}
$$

where we have $\boldsymbol{n}_{k}=\hat{\boldsymbol{U}}_{k n}^{\mathrm{H}} \boldsymbol{n}, \hat{\boldsymbol{U}}_{k n}^{\mathrm{H}}$ is a semi-unitary matrix and $\boldsymbol{n}_{k}$ has the same statistical properties as $\boldsymbol{n}$.

As seen in Fig. 1, after removing the MUI imposed on the $k$ th MS's signal at the RS with the aid of $T_{k}$, we can employ the linear weighting matrix $\boldsymbol{F}_{k}$ and the preprocessing matrix $\boldsymbol{P}_{k}$ at the RS for processing the signal vector $\hat{\boldsymbol{y}}_{k}$, resulting in the $R$-element signal vector $\tilde{\boldsymbol{x}}_{k}$ transmitted by the RS to the $k$ th $\mathrm{MS}$, which is given by

$$
\begin{aligned}
\tilde{\boldsymbol{x}}_{k} & =\boldsymbol{P}_{k} \boldsymbol{F}_{k} \hat{\boldsymbol{y}}_{k}=\boldsymbol{P}_{k} \boldsymbol{F}_{k} \hat{\boldsymbol{U}}_{k n}^{\mathrm{H}} \mathbf{y} \\
& =\boldsymbol{P}_{k} \boldsymbol{F}_{k} \hat{\boldsymbol{U}}_{k n}^{\mathrm{H}} \boldsymbol{H}_{k} \boldsymbol{x}_{k}+\boldsymbol{P}_{k} \boldsymbol{F}_{k} \boldsymbol{n}_{k}
\end{aligned}
$$

Consequently, the signal vector $\tilde{\boldsymbol{y}}_{k}$ at the receiver output of $k$ th
MS becomes

$$
\begin{aligned}
\tilde{\boldsymbol{y}}_{k} & =\tilde{\boldsymbol{H}}_{k} \sum_{i=1}^{K} \tilde{\boldsymbol{x}}_{k}+\tilde{\boldsymbol{n}}_{k} \\
& =\tilde{\boldsymbol{H}}_{k} \boldsymbol{P}_{k} \boldsymbol{F}_{k} \hat{\boldsymbol{y}}_{k}+\tilde{\boldsymbol{H}}_{k} \sum_{i=1, i \neq k}^{K} \boldsymbol{P}_{i} \boldsymbol{F}_{i} \hat{\boldsymbol{y}}_{i}+\tilde{\boldsymbol{n}}_{k}
\end{aligned}
$$

By appropriately choosing $\boldsymbol{P}_{k}$ of Fig. 1, the MUI $\tilde{\boldsymbol{H}}_{k} \sum_{i=1, i \neq k}^{K} \boldsymbol{P}_{i} \boldsymbol{F}_{i} \hat{\boldsymbol{y}}_{i}$ can be efficiently mitigated. The role of the weighting matrix $\boldsymbol{F}_{k}$ of Fig. 1 will be discussed later in Section 4.

To this end, we adopt the so-called BD-based multiuser transmission technique [12], where a new $\left(\sum_{i=1, i \neq k}^{K} N_{k} \times R\right)$-dimensional matrix $\breve{H}_{k}$ is generated for the $k$ th MS as

$$
\breve{\boldsymbol{H}}_{k}=\left[\tilde{\boldsymbol{H}}_{1}^{\mathrm{T}}, \ldots, \tilde{\boldsymbol{H}}_{k-1}^{\mathrm{T}}, \tilde{\boldsymbol{H}}_{k+1}^{\mathrm{T}}, \ldots, \tilde{\boldsymbol{H}}_{K}^{\mathrm{T}}\right]^{\mathrm{T}}
$$

Furthermore, if we assume that $R>\max \left(\sum_{i=1, i \neq k}^{K} N_{i}\right)$, then we have $\operatorname{rank}(\hat{\boldsymbol{H}}) \leq \sum_{i=1, i \neq k}^{K} N_{i}$. If the elements in each channel matrix are i.i.d, then we have $\operatorname{rank}\left(\widetilde{\boldsymbol{H}}_{k}\right)=\sum_{i=1, i \neq k}^{K} N_{i}$ with a probability of unity [12]. Furthermore, the SVD of the matrix $\breve{\boldsymbol{H}}_{k}$ is formulated as

$$
\breve{\boldsymbol{H}}_{k}=\breve{\boldsymbol{U}}_{k}\left[\breve{\boldsymbol{\Lambda}}_{k}^{1 / 2} \mathbf{0}\right]\left[\breve{\boldsymbol{V}}_{k s} \breve{\boldsymbol{V}}_{k n}\right]^{\mathrm{H}}, \quad k=1,2, \ldots, K
$$

where $\breve{\boldsymbol{\Lambda}}_{k}=\operatorname{diag}\left\{\breve{\lambda}_{1}, \breve{\lambda}_{2}, \ldots, \breve{\lambda}_{\sum_{i=1, i \neq k}^{K} N_{i}}\right\}$ contains the $\sum_{i=1, i \neq k}^{K} N_{i}$ non-zero eigenvalues of $\breve{H}_{k} \breve{H}_{k}$ or $\breve{\boldsymbol{H}}_{k} \breve{H}_{k}^{H}$. Furthermore, the columns of $\widetilde{\boldsymbol{U}}_{k}$ are constituted by the eigenvectors of $\breve{\boldsymbol{H}}_{k} \breve{\boldsymbol{H}}_{k}^{\mathrm{H}}, \breve{\boldsymbol{V}}_{k s}$ consists of the $\sum_{i=1, i \neq k}^{K} N_{i}$ eigenvectors corresponding to the signal subspace of $\breve{\boldsymbol{H}}_{k} \breve{\boldsymbol{H}}_{k}$, while $\breve{\boldsymbol{V}}_{k n}$ consists of the $\left(R-\sum_{i=1, i \neq k}^{K} N_{i}\right)$ eigenvectors corresponding to the null subspace of $\breve{\boldsymbol{H}}_{k} \breve{\boldsymbol{H}}_{k}$.

We can now determine the preprocessing matrix $P_{k}$ of Fig. 1 as a $\left(R \times J_{k}\right)$-component matrix, with $J_{k}=R-$ $\sum_{i=1, i \neq k}^{K} N_{i}$, where $P_{k}$ is given by

$$
\boldsymbol{P}_{k}=\breve{\boldsymbol{V}}_{k n}
$$

which is capable of completely removing the MUI term imposed on the $k$ th MS's received signal. Upon substituting (15) into (12), we arrive at

$$
\tilde{\boldsymbol{y}}_{k}=\boldsymbol{H}_{k 2} \boldsymbol{F}_{k} \boldsymbol{H}_{k 1} \boldsymbol{x}_{k}+\hat{\boldsymbol{n}}_{k}
$$

where the $\left(L_{k} \times M_{k}\right)$-dimensional matrix $\boldsymbol{H}_{k 1}$ is given by $\boldsymbol{H}_{k 1}=\hat{\boldsymbol{U}}_{k n}^{\mathrm{H}} \boldsymbol{H}_{k}$, while the $\left(N_{k} \times J_{k}\right)$-dimensional matrix $\boldsymbol{H}_{k 2}$ is formulated as $\boldsymbol{H}_{k 2}=\tilde{\boldsymbol{H}}_{k} \breve{\boldsymbol{V}}_{k n}$ and the $N_{k}$-component noise vector $\hat{\boldsymbol{n}}_{k}$ is given by $\hat{\boldsymbol{n}}_{k}=\boldsymbol{H}_{k 2} \boldsymbol{F}_{k} \boldsymbol{n}_{k}+\tilde{\boldsymbol{n}}_{k}$, which has a zero mean and a covariance matrix $\boldsymbol{R}_{k}$ of

$$
\boldsymbol{R}_{k}=E\left[\hat{\boldsymbol{n}}_{k} \hat{\boldsymbol{n}}_{k}^{\mathrm{H}}\right]=\sigma_{1}^{2} \boldsymbol{H}_{k 2} \boldsymbol{F}_{k} \boldsymbol{F}_{k}^{\mathrm{H}} \boldsymbol{H}_{k 2}^{\mathrm{H}}+\sigma_{2}^{2} \boldsymbol{I}_{N_{k}}
$$




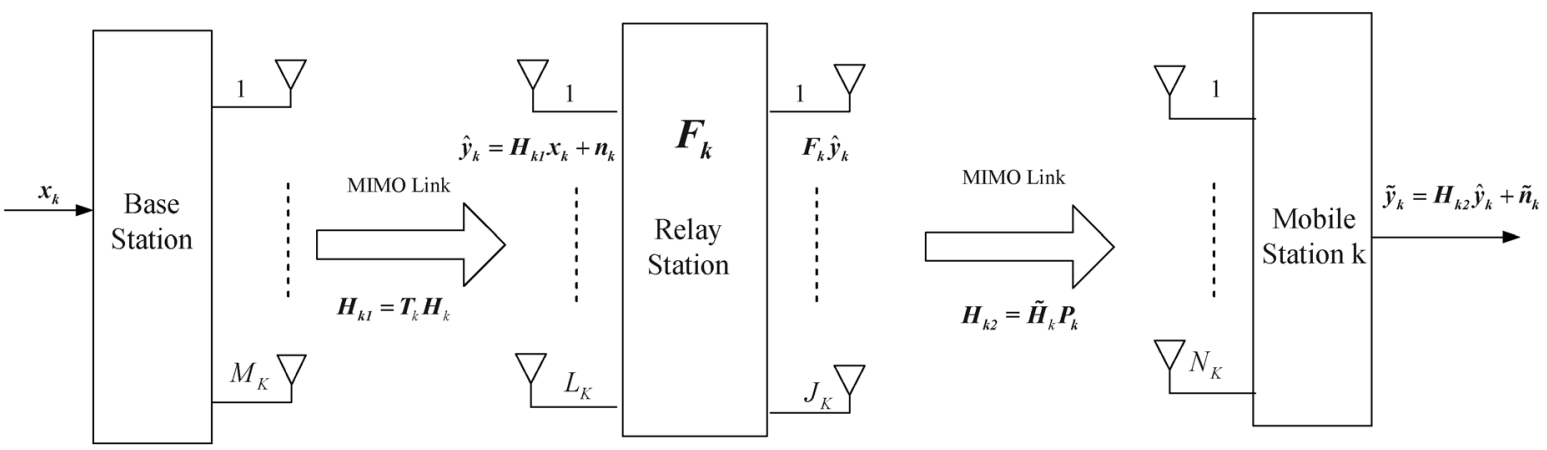

Fig. 2 Schematic representation of the effective single-user MIMO relay system with linear processing at RS and multiple antenna at each mobile

As we can see from (16), the MUI imposed on the $k$ th MS has been completely removed, hence the $k$ th MS effectively benefits from what is perceived to be a single-user MIMOaided relay system, which is the explicit benefit of using the linear weighting matrix $\boldsymbol{P}_{k}$ at the RS, as shown in Fig. 2.

Upon substituting (15) into (11) the signal vector $\tilde{\boldsymbol{x}}_{k}$ transmitted by the RS to the $k$ th MS becomes

$$
\begin{aligned}
\tilde{\boldsymbol{x}}_{k} & =\breve{\boldsymbol{V}}_{k n} \boldsymbol{F}_{k} \hat{\boldsymbol{y}}_{k}=\breve{\boldsymbol{V}}_{k n} \boldsymbol{F}_{k} \hat{\boldsymbol{U}}_{k n}^{\mathrm{H}} \boldsymbol{y}_{r} \\
& =\breve{\boldsymbol{V}}_{k n} \boldsymbol{F}_{k} \hat{\boldsymbol{U}}_{k n}^{\mathrm{H}} \boldsymbol{H}_{k} \boldsymbol{x}_{k}+\breve{\boldsymbol{V}}_{k n} \boldsymbol{F}_{k} \boldsymbol{n}_{k}
\end{aligned}
$$

Consequently, the composite multiuser signal vector $\boldsymbol{x}_{r}$ transmitted by the RS to the $K$ MSs becomes

$$
\boldsymbol{x}_{r}=\left[\tilde{\boldsymbol{x}}_{1}^{\mathrm{T}}, \ldots, \tilde{\boldsymbol{x}}_{K}^{\mathrm{T}}\right]^{\mathrm{T}}=\breve{\boldsymbol{V}} \boldsymbol{F} \hat{\boldsymbol{U}} \boldsymbol{y}
$$

where we have

$$
\begin{aligned}
\breve{\boldsymbol{V}} & =\left[\breve{\boldsymbol{V}}_{1 n}, \ldots, \breve{\boldsymbol{V}}_{K n}\right] \\
\boldsymbol{F} & =\operatorname{diag}\left[\boldsymbol{F}_{1}, \ldots, \boldsymbol{F}_{K}\right] \\
\hat{\boldsymbol{U}} & =\left[\hat{\boldsymbol{U}}_{1 n}, \ldots, \hat{\boldsymbol{U}}_{K n}\right]^{\mathrm{H}}
\end{aligned}
$$

Based on (19), we can see that the linear processing matrix $\boldsymbol{W}$ is given by

$$
\boldsymbol{W}=\breve{V} \boldsymbol{F} \hat{U}
$$

Having determined $\breve{\boldsymbol{V}}$ and $\hat{\boldsymbol{U}}$, let us now proceed by determining the $\left(J_{k} \times L_{k}\right)$-dimensional matrix $\boldsymbol{F}_{k}$. Depending on the specific optimisation criterion employed, $\boldsymbol{F}_{k}$ may have different realisations. Specifically, our goal is to determine that particular matrix $\boldsymbol{F}_{k}$, which maximises the achievable system capacity.

\section{Optimal linear weighting matrix}

The optimal linear weighting matrix, which maximises the achievable capacity has been provided in [7] for a singleuser MIMO aided relay system. However, the solution of [7] is limited to a square-shaped linear weighting matrix. In this section, we derive the canonical form of the linear weighting matrix for arbitrary dimensions.

Based on (16), the achievable capacity $C_{k}$ of the $k$ th MS for a given power $P_{k}$ allocated to the signal $\tilde{\boldsymbol{x}}_{k}$ transmitted by the
$\mathrm{RS}$ is formulated as [7]

$$
C_{k}=\log _{2}\left|\boldsymbol{I}_{M_{k}}+\frac{P_{1}}{M}\left(\boldsymbol{H}_{k 2} \boldsymbol{F}_{k} \boldsymbol{H}_{k 1}\right)^{\mathrm{H}} \boldsymbol{R}_{k}^{-1}\left(\boldsymbol{H}_{k 2} \boldsymbol{F}_{k} \boldsymbol{H}_{k 1}\right)\right|
$$

Upon invoking (18), the power constraint may be formulated as

$$
\operatorname{trace}\left(\tilde{\boldsymbol{x}}_{k} \tilde{\boldsymbol{x}}_{k}^{\mathrm{H}}\right)=\sigma_{1}^{2} \operatorname{trace}\left[\left(\boldsymbol{F}_{k}\left(\boldsymbol{I}_{L_{k}}+\rho_{1} \boldsymbol{H}_{k 1} \boldsymbol{H}_{k 1}^{\mathrm{H}}\right) \boldsymbol{F}_{k}^{\mathrm{H}}\right)\right] \leq P_{k}
$$

where $\rho_{1}=P_{1} / M \sigma_{1}^{2}$.

As usual, the eigenvalue decomposition of $\boldsymbol{H}_{k_{1}} \boldsymbol{H}_{k_{1}}^{\mathrm{H}}$ and $\boldsymbol{H}_{k 2}^{\mathrm{H}} \boldsymbol{H}_{k 2}$ can be expressed, respectively, as

$$
\begin{aligned}
\boldsymbol{H}_{k 1} \boldsymbol{H}_{k 1}^{\mathrm{H}} & =\boldsymbol{U}_{k 1} \boldsymbol{\Lambda}_{k 1} \boldsymbol{U}_{k 1}^{\mathrm{H}} \\
\boldsymbol{H}_{k 2}^{\mathrm{H}} \boldsymbol{H}_{k 2} & =\boldsymbol{V}_{k 2} \boldsymbol{\Lambda}_{k 2} \boldsymbol{V}_{k 2}^{\mathrm{H}}
\end{aligned}
$$

where $\boldsymbol{\Lambda}_{k 1}=\operatorname{diag}\left[\alpha_{k 1}, \ldots, \alpha_{k L_{l}}\right]$ hosts the eigenvalues of $\boldsymbol{H}_{k 1} \boldsymbol{H}_{k 1}^{\mathrm{H}}$ arranged in non-increasing order, while $\boldsymbol{\Lambda}_{k 2}=\operatorname{diag}\left[\beta_{k 1}, \ldots, \beta_{k J_{k}}\right]$ represents the eigenvalues of $\boldsymbol{H}_{k 2}^{\mathrm{H}} \boldsymbol{H}_{k 2}$, also arranged in non-increasing order.

Assuming that the $\left(J_{k} \times L_{k}\right)$-dimensional matrix $\boldsymbol{G}_{k}$ is formulated as

$$
\boldsymbol{G}_{k}=\frac{\sigma_{1}}{\sigma_{2}} \boldsymbol{F}_{k}=\boldsymbol{V}_{k 2} \boldsymbol{X}_{k}\left(\boldsymbol{I}_{L_{k}}+\rho_{1} \boldsymbol{\Lambda}_{k 1}\right)^{-1 / 2} \boldsymbol{U}_{k 1}^{\mathrm{H}}
$$

where $\boldsymbol{X}_{k}$ is an arbitrary $\left(J_{k} \times L_{k}\right)$-dimensional matrix, we can see that the optimum $\boldsymbol{X}_{k}$ must turn $\boldsymbol{X}_{k}^{\mathrm{H}} \boldsymbol{\Lambda}_{k 2} \boldsymbol{X}_{k}$ into a diagonal matrix [7], yielding

$$
\boldsymbol{X}_{k}^{\mathrm{H}} \boldsymbol{\Lambda}_{k 2} \boldsymbol{X}_{k}=\boldsymbol{\Lambda}_{k T}
$$

Based on (29), we have $\operatorname{rank}\left(\boldsymbol{\Lambda}_{k T}\right)=l_{k T} \leq \operatorname{rank}$ $\left(\boldsymbol{\Lambda}_{k 2}\right)=l_{k} \leq \min \left(J_{k}, N_{k}\right)$. Next we will discuss two different scenarios.

Scenario 1: Firstly, if $L_{k} \geq l_{k}$ is satisfied, we can partition $\boldsymbol{\Lambda}_{k 2}, \boldsymbol{X}_{k}$ and $\boldsymbol{\Lambda}_{k T}$ to ensure that

$$
\left[\begin{array}{ll}
\boldsymbol{X}_{k 1}^{\mathrm{H}} & \boldsymbol{X}_{k 2}^{\mathrm{H}}
\end{array}\right]\left[\begin{array}{cc}
\tilde{\boldsymbol{\Lambda}}_{k 2} & \mathbf{0} \\
\mathbf{0} & \mathbf{0}
\end{array}\right]\left[\begin{array}{l}
\boldsymbol{X}_{k 1} \\
\boldsymbol{X}_{k 2}
\end{array}\right]=\left[\begin{array}{cc}
\tilde{\boldsymbol{\Lambda}}_{k T} & \mathbf{0} \\
\mathbf{0} & \mathbf{0}
\end{array}\right]
$$

where $\tilde{\boldsymbol{\Lambda}}_{k 2}$ and $\tilde{\boldsymbol{\Lambda}}_{k T}$ are both $\left(l_{k} \times l_{k}\right)$-dimensional matrices, and $\tilde{\boldsymbol{\Lambda}}_{k 2}$ has a full rank, while $\tilde{\boldsymbol{\Lambda}}_{k T}$ does not necessarily have a full rank. Furthermore, $\boldsymbol{X}_{k 1}$ is a $\left(l_{k} \times L_{k}\right)$ dimensional matrix, while $\boldsymbol{X}_{k 2}$ is a $\left[\left(J_{k}-l_{k}\right) \times L_{k}\right]$ 
-dimensional matrix. Based on (30), we have

$$
\boldsymbol{X}_{k}=\left[\begin{array}{l}
\boldsymbol{X}_{k 1} \\
\boldsymbol{X}_{k 2}
\end{array}\right]=\left[\begin{array}{cc}
\tilde{\boldsymbol{\Lambda}}_{k 2}^{-1 / 2} & \boldsymbol{Q}_{k} \tilde{\boldsymbol{\Lambda}}_{k T}^{1 / 2} \\
& \boldsymbol{X}_{k 2}
\end{array}\right]
$$

where $\boldsymbol{Q}_{k}$ is an $\left(l_{k} \times l_{k}\right)$-dimensional unitary matrix, and $\hat{\boldsymbol{\Lambda}}_{k T}^{1 / 2}$ is an $\left(l_{k} \times L_{k}\right)$-dimensional matrix given by

$$
\hat{\boldsymbol{\Lambda}}_{k T}^{1 / 2}=\left[\hat{\boldsymbol{\Lambda}}_{k T}^{1 / 2} \mathbf{0}\right]
$$

Since $\boldsymbol{X}_{k 2}$ has no impact on $\boldsymbol{\Lambda}_{k T}$, we set $\boldsymbol{X}_{k 2}=\mathbf{0}$ to save power, which results in

$$
\begin{aligned}
\operatorname{trace}\left(\boldsymbol{X}_{k 1}^{\mathrm{H}} \boldsymbol{X}_{k 1}\right) & =\operatorname{trace}\left(\left(\hat{\boldsymbol{\Lambda}}_{k T}^{1 / 2}\right)^{\mathrm{H}} \boldsymbol{Q}_{k}^{\mathrm{H}} \tilde{\boldsymbol{\Lambda}}_{k 2}^{-1} \boldsymbol{Q}_{k} \hat{\boldsymbol{\Lambda}}_{k T}^{1 / 2}\right) \\
& =\operatorname{trace}\left(\boldsymbol{Q}_{k}^{\mathrm{H}} \tilde{\boldsymbol{\Lambda}}_{k 2}^{-1} \boldsymbol{Q}_{k} \tilde{\boldsymbol{\Lambda}}_{k T}\right) \geq \operatorname{trace}\left(\tilde{\boldsymbol{\Lambda}}_{k 2}^{-1} \tilde{\boldsymbol{\Lambda}}_{k T}\right)
\end{aligned}
$$

where the equality is achieved for $\boldsymbol{Q}_{k}=\boldsymbol{I}$. In (33), we exploited the facts that given any two $(N \times N)$-element positive semi-definite Hermitian matrices $\boldsymbol{A}$ and $\boldsymbol{B}$ with eigenvalues of $\lambda_{i}(A)$ and $\lambda_{i}(B)$ arranged in non-increasing order, respectively, we have [13]

$$
\sum_{i=1}^{N} \lambda_{i}(\boldsymbol{A}) \lambda_{N+1-i}(\boldsymbol{B}) \leq \operatorname{trace}(\boldsymbol{A B}) \leq \sum_{i=1}^{N} \lambda_{i}(\boldsymbol{A}) \lambda_{i}(\boldsymbol{B})
$$

Hence the optimal $\boldsymbol{X}_{k}$ of Scenario 1 is given by

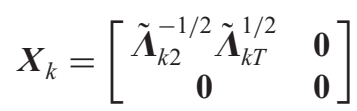

In summary, the optimal $\left(J_{k} \times L_{k}\right)$-dimensional matrix of $\boldsymbol{X}_{k}$ has the form

$$
\boldsymbol{X}_{k}=\left[\begin{array}{cc}
\hat{\boldsymbol{X}}_{k} & \mathbf{0} \\
\mathbf{0} & \mathbf{0}
\end{array}\right]
$$

where $\hat{\boldsymbol{X}}_{k}$ is an $\left(l_{k} \times l_{k}\right)$-dimensional diagonal matrix, given by $\hat{\boldsymbol{X}}_{k}=\operatorname{diag}\left[\hat{x}_{k 1}, \ldots, \hat{x}_{k l_{k}}\right]$.

Scenario 2: By contrast, if $L_{k}<l_{k}$, we can partition $\boldsymbol{\Lambda}_{k 2}$, $\boldsymbol{X}_{k}$ and $\boldsymbol{\Lambda}_{k T}$ to ensure that

$$
\left[\begin{array}{ll}
\boldsymbol{X}_{k 1}^{\mathrm{H}} & \boldsymbol{X}_{2 k}^{\mathrm{H}}
\end{array}\right]\left[\begin{array}{cc}
\tilde{\boldsymbol{\Lambda}}_{k 2} & \mathbf{0} \\
\mathbf{0} & \mathbf{0}
\end{array}\right]\left[\begin{array}{l}
\boldsymbol{X}_{k 1} \\
\boldsymbol{X}_{k 2}
\end{array}\right]=\left[\begin{array}{cc}
\tilde{\boldsymbol{\Lambda}}_{k T} & \mathbf{0} \\
\mathbf{0} & \mathbf{0}
\end{array}\right]
$$

where $\tilde{\boldsymbol{\Lambda}}_{k 2}$ and $\tilde{\boldsymbol{\Lambda}}_{k T}$ are $\left(l_{k} \times l_{k}\right)$-dimensional and $\left(l_{k T} \times l_{k T}\right)$ dimensional matrices, respectively, and both $\tilde{\boldsymbol{\Lambda}}_{k 2}$ and $\tilde{\boldsymbol{\Lambda}}_{k T}$ have a full rank. Furthermore, $\boldsymbol{X}_{k 1}$ is an $\left(l_{k} \times L_{k}\right)$ dimensional matrix, while $\boldsymbol{X}_{k 2}$ is a $\left[\left(J_{k}-l_{k}\right) \times L_{k}\right]$ dimensional matrix. Based on (37), we have

$$
\boldsymbol{X}_{k}=\left[\begin{array}{l}
\boldsymbol{X}_{k 1} \\
\boldsymbol{X}_{k 2}
\end{array}\right]=\left[\begin{array}{cc}
\tilde{\boldsymbol{\Lambda}}_{k 2}^{-1 / 2} & \boldsymbol{Q}_{k} \tilde{\boldsymbol{\Lambda}}_{k T}^{1 / 2} \\
\boldsymbol{X}_{k 2}
\end{array}\right]
$$

where $\boldsymbol{Q}_{k}^{H}$ is an $\left(l_{k T} \times l_{k}\right)$-dimensional semi-unitary matrix, and $\hat{\Lambda}_{k T}^{1 / 2}$ is an $\left(l_{k T} \times L_{k}\right)$-dimensional matrix given by

$$
\hat{\boldsymbol{\Lambda}}_{k T}^{1 / 2}=\left[\hat{\boldsymbol{\Lambda}}_{k T}^{1 / 2} \mathbf{0}\right]
$$

Similarly, we set $\boldsymbol{X}_{k 2}=\mathbf{0}$ to save power and hence we have

$$
\begin{aligned}
\operatorname{trace}\left(\boldsymbol{X}_{k 1}^{\mathrm{H}} \boldsymbol{X}_{k 1}\right) & =\operatorname{trace}\left(\tilde{\boldsymbol{\Lambda}}_{k 2}^{-1} \boldsymbol{Q}_{k} \tilde{\boldsymbol{\Lambda}}_{k T} \boldsymbol{Q}_{k}^{\mathrm{H}}\right) \\
& \geq \operatorname{trace}\left(\tilde{\boldsymbol{\Lambda}}_{k 2} \tilde{\boldsymbol{\Lambda}}_{k T}\right)
\end{aligned}
$$

where $\breve{\boldsymbol{\Lambda}}_{k 2}$ is an $\left(l_{k T} \times l_{k T}\right)$-dimensional submatrix of $\tilde{\boldsymbol{\Lambda}}_{k 2}^{-1}$, composed of the first $l_{k T}$ rows and $l_{k T}$ columns of $\tilde{\Lambda}_{k 2}^{-1}$ and the equality in (40) is achieved, when $\boldsymbol{Q}_{k}$ equals the first $l_{k T}$ columns of $\boldsymbol{I}_{l_{k}}$ according to (34).

Hence the optimal $\boldsymbol{X}_{k}$ of Scenario 2 is given by

$$
\boldsymbol{X}_{k}=\left[\begin{array}{cc}
\breve{\boldsymbol{\Lambda}}_{k 2} \tilde{\boldsymbol{\Lambda}}_{k T}^{1 / 2} & \mathbf{0} \\
\mathbf{0} & \mathbf{0}
\end{array}\right]
$$

In summary, the optimal $\left(J_{k} \times L_{k}\right)$-dimensional matrix of $\boldsymbol{X}_{k}$ has the form

$$
\boldsymbol{X}_{k}=\left[\begin{array}{c}
\hat{\boldsymbol{X}}_{k} \\
\mathbf{0}
\end{array}\right]
$$

where $\hat{\boldsymbol{X}}_{k}$ is an $\left(L_{k} \times L_{k}\right)$-element diagonal matrix, which is given by $\hat{\boldsymbol{X}}_{k}=\operatorname{diag}\left[\hat{x}_{k 1}, \ldots, \hat{x}_{k L_{k}}\right]$.

By combining Scenarios 1 and 2 discussed above, we conclude that the optimal $\left(J_{k} \times L_{k}\right)$-dimensional matrix $\boldsymbol{X}_{k}$ has the form

$$
\boldsymbol{X}_{k}=\left[\begin{array}{cc}
\hat{\boldsymbol{X}}_{k} & \mathbf{0} \\
\mathbf{0} & \mathbf{0}
\end{array}\right]
$$

where $\hat{\boldsymbol{X}}_{k}$ is an $\left(l_{k}^{\prime} \times l_{k}^{\prime}\right)$-dimensional diagonal matrix, and $l_{k}^{\prime}=\min \left\{L_{k}, l_{k}\right\}$, which is given by $\hat{\boldsymbol{X}}_{k}=\operatorname{diag}\left[\hat{x}_{k 1}, \ldots, \hat{x}_{k l^{\prime}}\right]$.

Based on (25), the optimal weighting matrix $\boldsymbol{F}_{k}$ is given by

$$
\boldsymbol{F}_{k}=\boldsymbol{V}_{k 2} \boldsymbol{\Lambda}_{k F} \boldsymbol{U}_{k 1}^{\mathrm{H}}=\boldsymbol{V}_{k 2}\left[\begin{array}{cc}
\tilde{\boldsymbol{\Lambda}}_{k} & \mathbf{0} \\
\mathbf{0} & \mathbf{0}
\end{array}\right] \boldsymbol{U}_{k 1}^{\mathrm{H}}
$$

where we have $\boldsymbol{\Lambda}_{k F}=\left[\begin{array}{cc}\tilde{\boldsymbol{\Lambda}}_{k} & \mathbf{0} \\ \mathbf{0} & \mathbf{0}\end{array}\right]$, and $\tilde{\boldsymbol{\Lambda}}_{k}$ is an $\left(l_{k}^{\prime} \times l_{k}^{\prime}\right)$ dimensional diagonal matrix, given by $\tilde{\boldsymbol{\Lambda}}_{k}=\operatorname{diag}\left[\tilde{\lambda}_{k 1}, \ldots\right.$, $\tilde{\lambda}_{k l_{k}^{\prime}}$, where the powerq allocation coefficients $\tilde{\lambda}_{k i}$ are given by [7] (see (45))

Furthermore, in (45), $v$ should be chosen to meet [7]

$$
\sum_{k=1}^{K} \sigma_{2}^{2} \sum_{i=1}^{l_{k}^{\prime}} \hat{x}_{k i}^{2}-P_{r} \leq 0
$$

\section{Simulation results}

In this section, our simulation results are provided. The notation of $\left(M-R-N_{k}-K\right)$ is used in the figures to

$$
\tilde{\lambda}_{k i}=\frac{\sigma_{2}}{\sigma_{1}} \sqrt{\frac{1}{2 \beta_{k i}\left(1+\rho_{1} \alpha_{k i}\right)}\left[\sqrt{\left(\rho_{1} \alpha_{k i}\right)^{2}+4 \rho_{1} \alpha_{k i} \beta_{k i} \frac{1}{v \sigma_{2}^{2} \ln 2}}-\rho_{1} \alpha_{k i}-2\right]^{+}}
$$




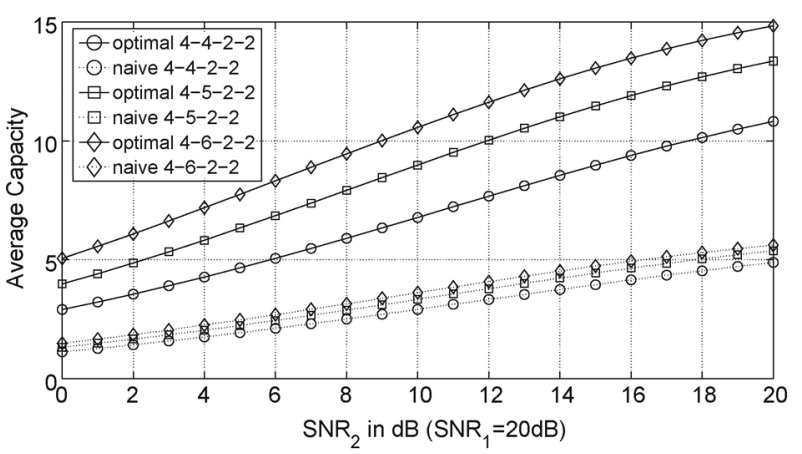

Fig. 3 Average capacity against the average $S N R_{2}$ when $S N R_{1}$ is fixed at $20 \mathrm{~dB}$ for two MSs

Notation of $\left(M-R-N_{k}-K\right)$ is used in the figures to characterise the antenna configurations. Furthermore, for a given $M, M_{k}=M / K$ is used

characterise the antenna configurations. Furthermore, for a given $M, M_{k}=M / K$ is used. Moreover, we define the parameters $\mathrm{SNR}_{1}$ and $\mathrm{SNR}_{2}$, respectively, as

$$
\mathrm{SNR}_{1}=\frac{P_{s} / M}{\sigma_{1}^{2}}=\frac{1}{\sigma_{1}^{2}}
$$

where we exploited the property of $P_{s}=E\left[x x^{H}\right]=M$ and

$$
\mathrm{SNR}_{2}=\frac{P_{r} / R}{\sigma_{2}^{2}}=\frac{1}{\sigma_{2}^{2}}
$$

assuming that $P_{r}=R$. Furthermore, the entries in $\boldsymbol{H}$ and $\tilde{\boldsymbol{H}}_{k}$ are assumed to be i.i.d. complex Gaussian variables with zero means and unity covariance.

Moreover, the so-called naive weighting matrix is used as the benchmark. Specifically, for naive weighting matrix, $\boldsymbol{F}_{k}$ is chosen to be $\boldsymbol{F}_{k}=\beta \boldsymbol{I}_{\left(J_{k}, L_{k}\right)}$, where $\boldsymbol{I}_{\left(J_{k}, L_{k}\right)}$ is a $\left(J_{k} \times L_{k}\right)$ dimensional matrix with $1 \mathrm{~s}$ on its diagonal and zeros elsewhere. Additionally, the $\beta$ is the power control factor, which can be determined by $\operatorname{Tr}\left(\boldsymbol{x}_{r} \boldsymbol{x}_{r}^{\mathrm{H}}\right)=P_{r}$.

In Fig. 3 , the average capacity against the average $\mathrm{SNR}_{2}$ is plotted, when $\mathrm{SNR}_{1}$ was fixed at $20 \mathrm{~dB}$. Furthermore, the number of DL transmit antennas at the BS is set to $M=4$ and the number of MSs is set to $K=2$, each equipped with $N_{k}=2$ antennas. In this case, the matrix $\boldsymbol{F}_{k}$ is a square one. As we can see from Fig. 3 that our optimal weighting matrix substantially outperforms the naive one for any system configurations. A similar conclusion may be inferred from Fig. 4, when the role of $\mathrm{SNR}_{1}$ and $\mathrm{SNR}_{2}$ was swapped, that is, we had $\mathrm{SNR}_{2}=20 \mathrm{~dB}$.

In Fig. 5, the average capacity against the average $\mathrm{SNR}_{2}$ is quantified, when $\mathrm{SNR}_{1}$ is fixed at $20 \mathrm{~dB}$. Furthermore, the number of DL transmit antennas at the BS is set to $M=4$ and the number of receive antennas at the RS is assumed to take the values of $R=4,5,6$. Moreover, the number of MSs is set to $K=2$, each equipped with $N_{k}=3$ receive antennas. In this case, the matrix $\boldsymbol{F}_{k}$ is a non-square one. As we can see from Fig. 5, for a given antenna configuration at the BS and RS, the capacity achieved by the optimal weighting matrix substantially outperforms that by naive one. Again, similar trends may also be observed from Fig. 6, where the role of $\mathrm{SNR}_{1}$ and $\mathrm{SNR}_{2}$ was swapped, that is, we had $\mathrm{SNR}_{2}=20 \mathrm{~dB}$.

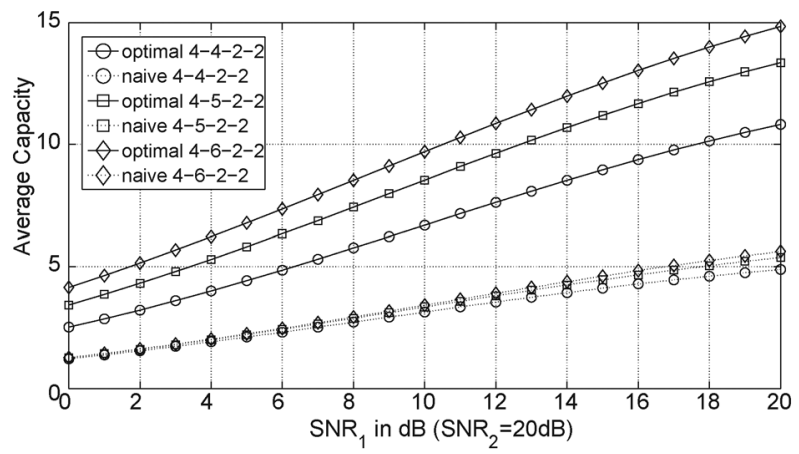

Fig. 4 Average capacity against the average $S N R_{1}$ when $S N R_{2}$ is fixed at $20 \mathrm{~dB}$

Notation of $\left(M-R-N_{k}-K\right)$ is used in the figures to characterise the antenna configurations. Furthermore, for a given $M, M_{k}=M / K$ is used

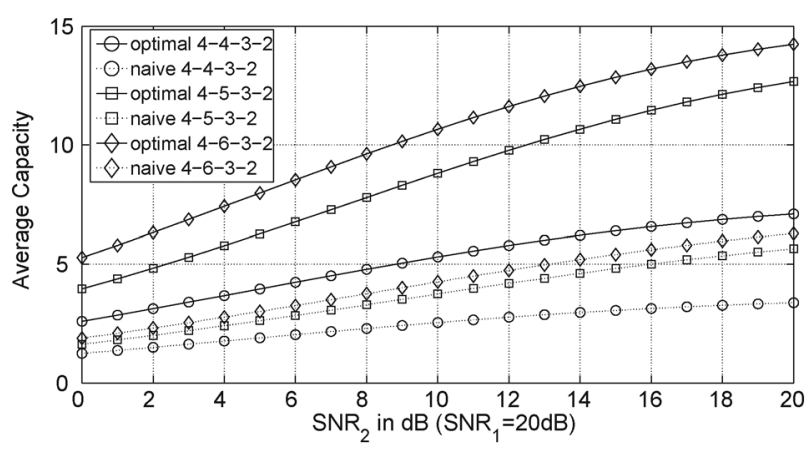

Fig. 5 Average capacity against the average $S N R_{2}$ when $S N R_{1}$ is fixed at $20 \mathrm{~dB}$ for three MSs

Notation of $\left(M-R-N_{k}-K\right)$ is used in the figures to characterise the antenna configurations. Furthermore, for a given $M, M_{k}=M / K$ is used

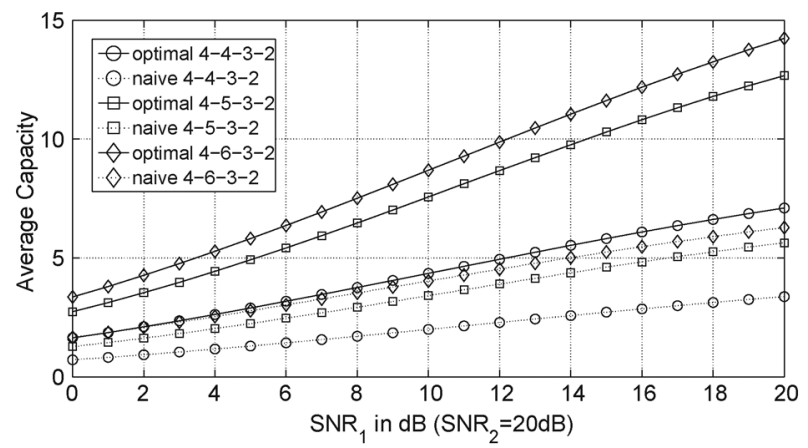

Fig. 6 Average capacity against the average $\mathrm{SNR}_{1}$ when $\mathrm{SNR}_{2}$ is fixed at $20 \mathrm{~dB}$

Notation of $\left(M-R-N_{k}-K\right)$ is used in the figures to characterise the antenna configurations. Furthermore, for a given $M, M_{k}=M / K$ is used

\section{Conclusions}

In this paper, we proposed a block-diagonalisation based multiuser MIMO-aided relaying scheme using linear processing at the RS. The proposed algorithm decomposes a multiuser MIMO-aided relay scheme into several parallel single-user MIMO relay schemes without imposing any requirements at the BS. Furthermore, the optimal linear matrix designed for maximum capacity is also derived in this paper, which significantly outperforms the so-called naive weighting matrix. 


\section{www.ietdl.org}

\section{Acknowledgments}

The financial support of National Basic Research Program of China (973 Program) (2009CB320404), of the National Natural Science Foundation of China (60972047), of ISN1003002, of Innovation Fund for returned overseas Scholars of Xidian University (64101879), and of the China 111 Project (B08038), of National Science and Technology Major Project (2011ZX03005-003-03, 2011ZX03005-004, 2011ZX03004-003), as well as that of the RCUK under the UK-China Science Bridge Initiative in $4 \mathrm{G}$ wireless communications are gratefully acknowledged.

\section{References}

1 Telatar, I.E.: 'Capacity of multi-antenna Gaussian channels', Eur. Trans. Telecommun., 1999, 10, pp. 585-595

2 Wolniansky, P.W., Foschini, G.J., Golden, G.D., Valenzuela, R.A.: 'V-BLAST: an architecture for realizing very high data rates over the rich-scattering wireless channel'. Int. Symp. on Signals, Systems, and Electronics, Pisa, Italy, 29 September-2 October 1998, pp. 295-300

3 Alamouti, S.M.: 'A simple transmit diversity technique for wireless communications', IEEE J. Sel. Areas Commun., 1998, 16, pp. 1451-1458
4 Laneman, J.N., Tse, D.N., Wornell, G.W.: 'Cooperative diversity in wireless networks: efficient protocols and outage behavior', IEEE Trans. Inf. Theory, 2004, 50, pp. 3062-3080

5 Wang, B., Zhang, J., Host-Madsen, A.: 'On the capacity of MIMO relay channels', IEEE Trans. Inf. Theory, 2005, 51, pp. 29-43

6 Munoz-Medina, O., Vidal, J., Agustin, A.: 'Linear transceiver design in nonregenerative relays with channel state information', IEEE Trans. Signal Process., 2007, 55, pp. 2593-2604

7 Tang, X., Hua, Y.: 'Optimal design of non-regenerative MIMO wireless relays', IEEE Trans. Wirel. Commun., 2007, 6, pp. 1398-1407

8 Guan, W., Luo, H.: 'Joint MMSE transceiver design in non-regenerative MIMO relay systems', IEEE Commun. Lett., 2008, 7, pp. 517-519

9 Zhang, G., Li, G., Qin, J.: 'Fast antenna subset selection algorithms for multiple-input multiple-output relay systems', IET Commun., 2009, 3, (11), pp. $1693-1703$

10 Zhang, R., Chai, C.C., Liang, Y.C.: 'Joint beamforming and power control for multiantenna relay broadcast channel with QoS constraints', IEEE Trans. Signal Process., 2009, 57, pp. 726-737

11 Chae, C.B., Tang, T., Heath, R.W., Cho, S.: 'MIMO relaying with linear processing for multiuser transmission in fixed relay networks', IEEE Trans. Signal Process., 2008, 56, pp. 727-738

12 Choi, L., Murch, R.D.: 'A transmit preprocessing technique for multiuser MIMO systems using a decomposition approach', IEEE Trans. Wirel. Commun., 2004, 3, pp. 20-24

13 Horn, R.A., Johnson, C.R.: 'Matrix analysis' (Cambridge University Press, 1985) 